\title{
Three-dimensional evaluation of ductal tissue in coarctation of the aorta using $X$-ray phase-contrast tomography
}

\author{
Ryuma Iwaki, MD, ${ }^{\mathrm{a}}$ Hironori Matsuhisa, $\mathrm{MD}, \mathrm{PhD},{ }^{\mathrm{a}}$ Masato Hoshino, $\mathrm{PhD},{ }^{\mathrm{b}}$ and \\ Yoshihiro Oshima, MD, PhD, ${ }^{\text {a }}$ Kobe and Sayo, Japan
}

From the a Department of Cardiovascular Surgery, Kobe Children's Hospital, Kobe; 'Japan Synchrotron Radiation Research Institute (SPring-8), Sayo, Japan.

This study was approved by the SPring-8 Proposal Review Committee (2014B1306, 2015A1435, 2015B1370). It was supported by JSPS KAKENHI grant number $15 \mathrm{~K} 15505$ and 26293249 . This research was partially supported by the Japan Agency for Medical Research and Development.

Disclosures: Authors have nothing to disclose with regard to commercial support.

Received for publication March 2, 2016; revisions received May 5, 2016; accepted for publication May 27, 2016; available ahead of print July 9, 2016.

Address for reprints: Ryuma Iwaki, MD, Department of Cardiovascular Surgery, Kobe Children's Hospital, 1-1-1, Takakura-dai, Suma-ku, Kobe 654-0081, Japan (E-mail: iwaki_kch@hp.pref.hyogo.jp).

J Thorac Cardiovasc Surg 2016;152:1454-6

$0022-5223 / \$ 36.00$

Copyright $@ 2016$ by The American Association for Thoracic Surgery

http://dx.doi.org/10.1016/j.jtcvs.2016.05.053

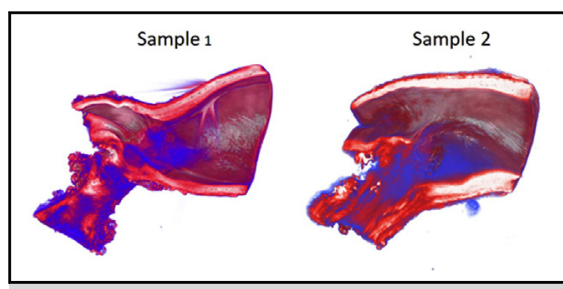

$3 \mathrm{D}$ extent of ductal tissue with $3 \mathrm{D}$ reconstruction of phase-contrast tomography images.

Central Message
Evaluation of three-dimensional extent of
ductal tissue with resected ductal segments of
coarctation of the aorta using X-ray phase-
contrast tomography.
See Editorial Commentary page
1457 .

Two samples of CoA obtained during surgery at Kobe Children's Hospital were used. During the surgery, the aortic segment including the hypoplastic isthmus, the ductus arteriosus (DA), and a wide margin of descending aorta beyond the insertion of the DA was excised in an en bloc fashion. These samples were fixed in $10 \%$ formalin solution, and 3D images were obtained using an XPCT imaging system based on the Talbot grating interferometer at SPring-8 (Hyogo, Japan). After scanning, the samples were histologically evaluated by Elastica van Gieson (EVG) staining.

The XPCT sectional images clearly demonstrated the distribution of ductal media as a low-density area. The extent of low-density intimal thickness, which can be the ductal intima, was also clearly demonstrated (Figure 2, A). The extent of the ductal tissue in XPCT images was consistent with the histologic findings from the EVG staining procedure (Figure 2, B). For quantitative analysis, histograms of the mass density map of the aortic media are shown in Figure 2, $C$. The densities of the aortic media, ductal media, and intima were

Scanning this QR code will take you to a video for the article.

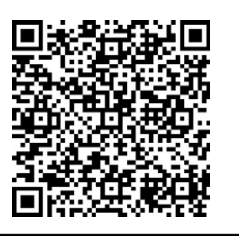



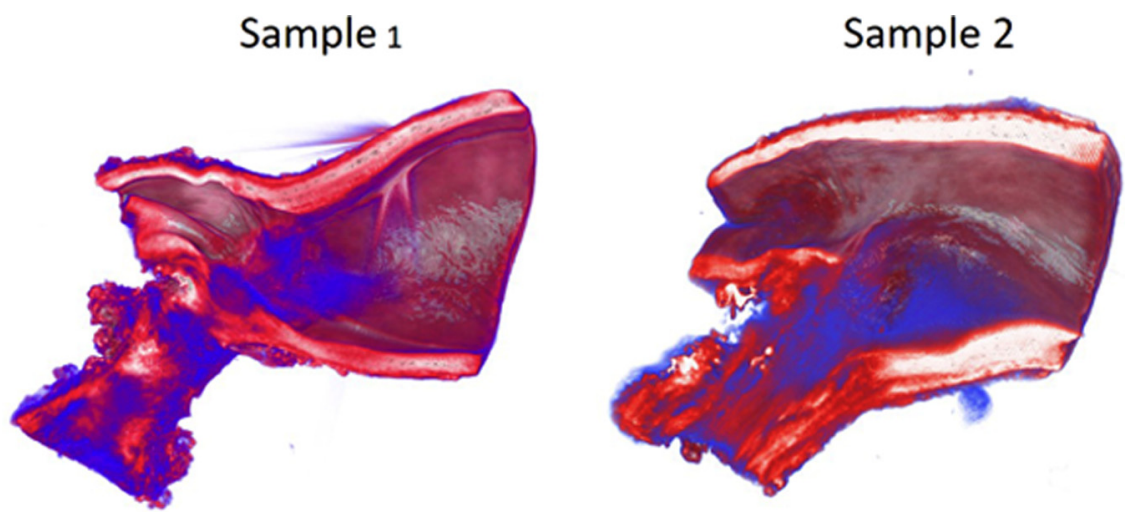

FIGURE 1. Three-dimensional extent of ductal tissue with 3-demensional reconstruction of phase-contrast tomography images.

around $1.08 \mathrm{~g} / \mathrm{cm}^{3}, 1.06 \mathrm{~g} / \mathrm{cm}^{3}$, and $1.04 \mathrm{~g} / \mathrm{cm}^{3}$, respectively. The segmented 3D images were constructed using Drishti (http://sf.anu.edu.au/Vizlab/drishti/index.shtml) (Video 1). The aortic media that contained elastic fibers is shown in white (high density), and the ductal media is shown in red (low density). The images clearly demonstrate the 3D extent of the ductal intima (blue, very low density). On the ductal side, the ductal intima extended distally beyond the border of the ductal media. In sample 1, the ductal intima was confirmed in the aorta opposite the ductal insertion. However, it was not observed in sample 2.
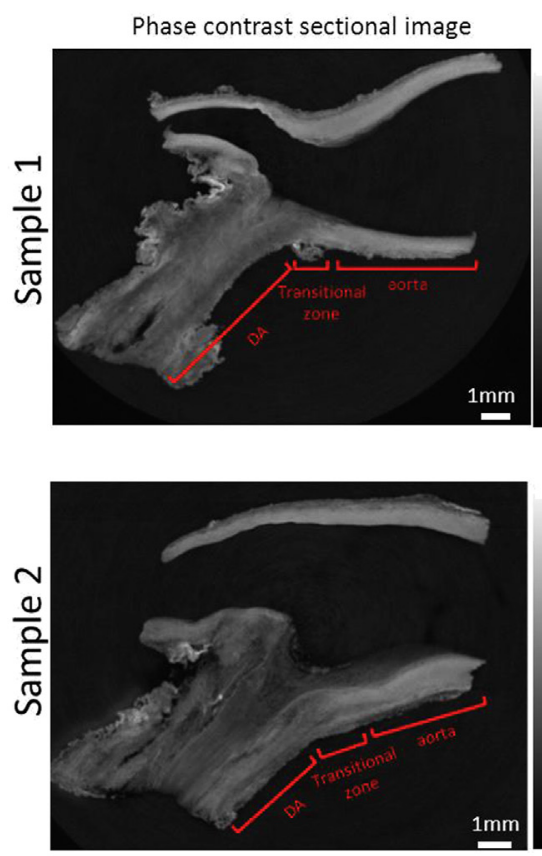

A

FIGURE 2. Identification of the ductal tissue and the aortic wall tissue by comparing X-ray phase-contrast tomographic images with images captured using Elastica van Gieson $(E V G)$ staining. A, Longitudinal sectional images of X-ray phase-contrast tomography clearly demonstrate the ductal media and intima as low-density areas and the normal aortic media as a high-density area. The bars on the right indicate the density scale. B, Corresponding histologic sections of EVG stain (upper, $\times 40$; lower, $\times 400$ ). The media of the ductus arteriosus $(D A)$ largely consists of smooth muscle cells, instead of circumferentially arranged layers of elastic fibers. The media of the aorta is composed of parallel elastic fibers. The intimal thickness, which can be the ductal intima (intimal cushion), extends beyond the border between the normal aorta and ductal arteriosus at the media. C, Mass density histograms from the ductal media to the descending aortic (decAo) media.
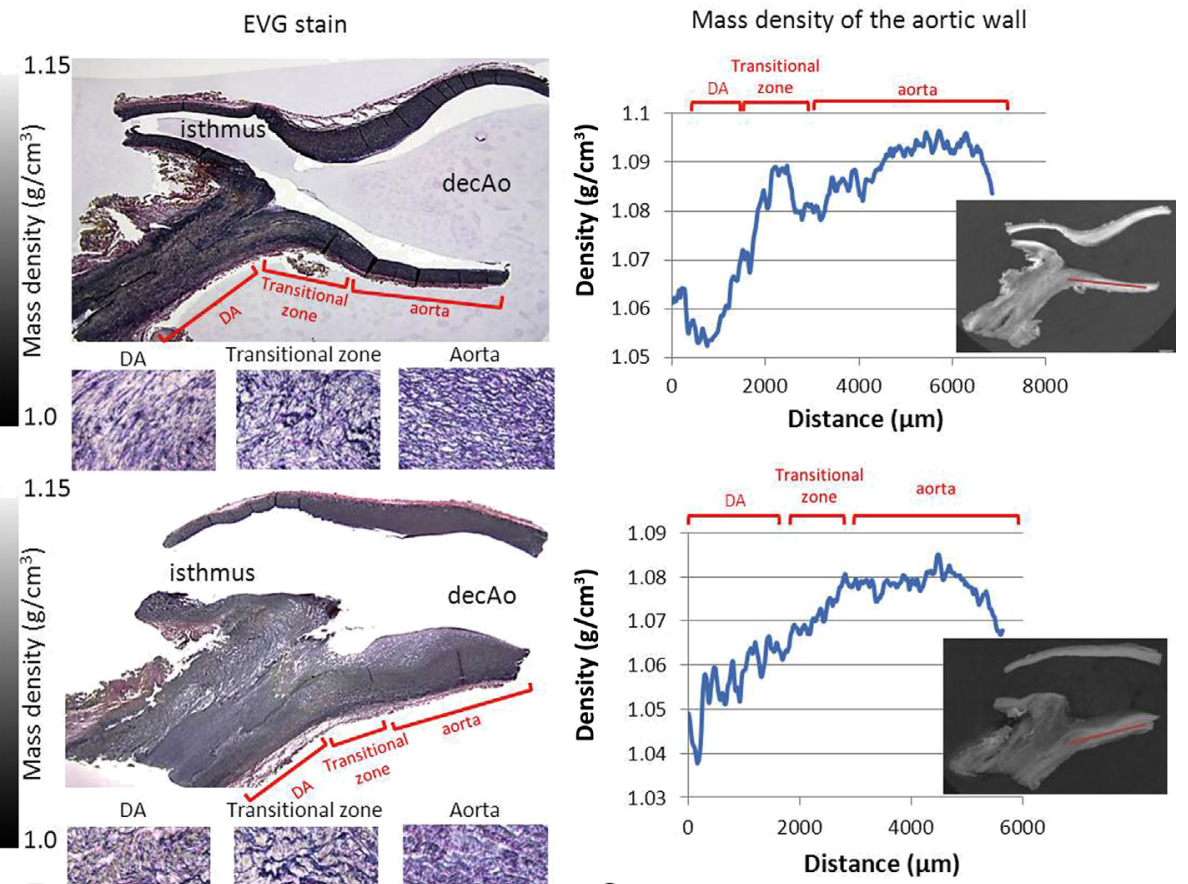

C

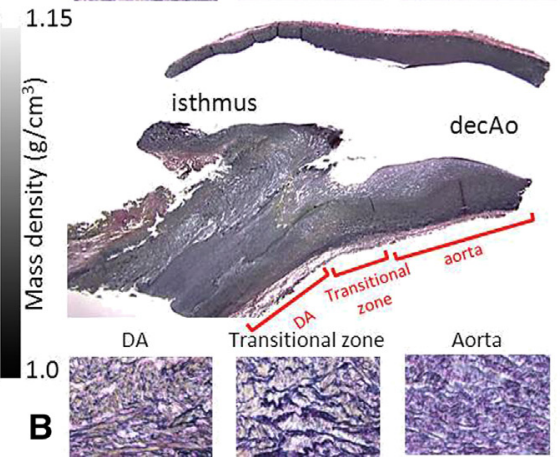




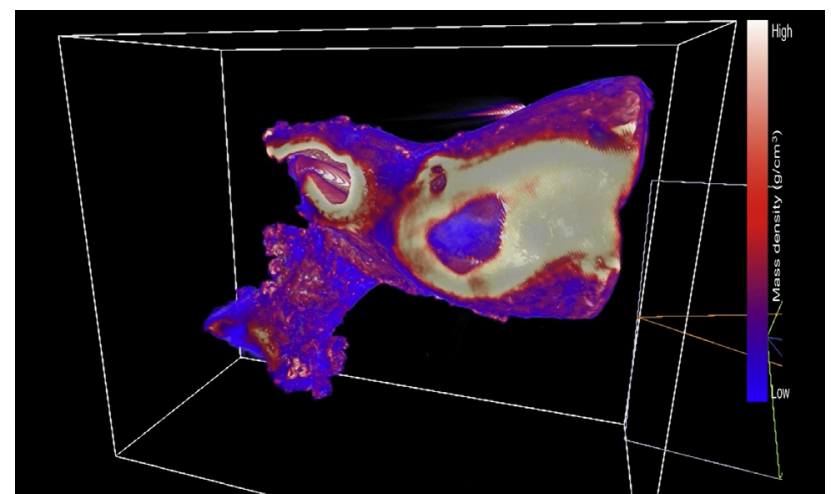

VIDEO 1. Three-dimensional reconstruction of phase-contrast tomography images. The aortic media containing elastic fibers is represented in white (high density), and media and intima of the ductal arteriosus are represented in red (low density) and blue (lowest density), respectively. Video available at: http://www.jtcvsonline.org/article/S0022-5223(16) 30491-3/addons.

In this study, the contrast resolution of XPCT images was comparable with that obtained in histologic assessments with EVG staining. From the 3D images, the ductal intima spread more distally and laterally than the ductal media, especially on the inner curvature. In addition, incomplete resection of the ductal intima was suspected in sample 2. Extensive resection of the inner curvature rather than the greater curvature may minimize the risk of recoarctation. However, it has not been elucidated which layer of remnant ductal tissue (intima or media) is mainly responsible for recoarctation. To clarify the cause of recoarctation, further investigation of CoA samples and careful postoperative observation are essential.

The clinical application of XPCT is gaining attention; however, imaging of the in vivo heart is challenging. In the present circumstances, detection of phase-shift values in vivo is more difficult compared with using isolated samples. Future development of the XPCT system for in vivo human heart imaging can be expected.

We would like to thank Drs Kiyozo Morita, Yukihiro Kaneko, Masashi Takahashi, Takuro Tsukube, Susumu Minamisawa, and Yutaka Okita for giving insightful comments and suggestions; Dr Makiko Yoshida for assisting with the photomicrographs; and Jyunko Kawabata, Chikako Yonetani, and Masayo Yamagishi for their excellent technical assistance.

\section{References}

1. Russell GA, Berry PJ, Watterson K, Dhasmana JP, Wisheart JD. Patterns of ductal tissue in coarctation of the aorta in the first three months of life. J Thorac Cardiovasc Surg. 1991;102:596-601.

2. Hoshino M, Uesugi K, Tsukube T, Yagi N. Quantitative and dynamic measurements of biological fresh samples with X-ray phase contrast tomography. J Synchrotron Radiat. 2014;21:1347-57.

3. Tsukube T, Yagi N, Hoshino M, Nakashima Y, Nakagawa K, Okita Y. Impact of synchrotron radiation-based X-ray phase-contrast tomography on understanding various cardiovascular surgical pathologies. Gen Thorac Cardiovasc Surg. 2015; 63:590-2. 